MINI-SYMPOSIUM

\title{
Pericardial effusion: haemodynamic spectrum
}

\author{
R Shabetai
}

Heart 2004;90:255-256. doi: 10.1136/hrt.2003.024810

$\mathrm{H}$ aemodynamic abnormalities, caused by pericardial effusion, range from undetectable or mild, to life threatening, depending on the determinants discussed below. These include the rate at which the effusion accumulates and whether or not the pericardium is scarred and thus adds an element of constrictive pericarditis. Pericardial adhesions or organisation of the fluid can result in localised, and thus atypical, tamponade. Pericardial effusion is the cause of a number of distinct clinical and haemodynamic syndromes.

Fundamental to understanding their pathophysiology are the biomechanical properties of the pericardium. The pressure-volume relation of normal pericardium, after an initial short shallow portion that allows the pericardium to stretch slightly in response to physiological events, such as changes in posture or volume status, shows a minimal increase in pericardial pressure. Thereafter, the pressure increase is extremely steep. This $\mathrm{J}$ shaped curve $\mathrm{e}^{1}$ indicates that a sudden increase in the volume of pericardial fluid can slightly stretch the pericardium, and thus an increase of as little as 100 or $200 \mathrm{ml}$ may elevate pericardial pressure from its normal ambient, or slightly sub-ambient value, to $30 \mathrm{~mm} \mathrm{Hg}$ or more, defining severe cardiac tamponade.

At the other extreme, a pericardial effusion developing over several weeks or months may reach a volume measured in litres accompanied by a considerably more modest increase in pericardial pressure. ${ }^{2}$ The explanation for this difference is that the response of the pericardium to gradual stretch differs from its response to acute stretch. A slower accumulation of pericardial fluid causes pericardial compliance to increase; its pressure-volume curve is shifted to the right and the slope of the steep portion is greatly reduced, such that for a given increment in volume, the pressure rise is appreciably less. ${ }^{3}$ This is the tamponade usually encountered in medical clinics and wards, whereas acute tamponade is found in interventional cardiac laboratories, as a consequence of puncture of a cardiac chamber or a vessel, in trauma units to which victims of mediastinal injury are admitted, and in intensive care units where acute tamponade is a sequel to rupture of a myocardial infarction or aortic aneurysm. Because the pressure-volume relation is curved, compliance decreases with increasing volume. Even in "medical tamponade" pericardial pressure may rise to $30 \mathrm{~mm} \mathrm{Hg}$ as fluid continues to accumulate faster than it can be absorbed. However, this elevation occurs at much greater volume of pericardial fluid compared with acute tamponade. The volume of effusion at which this development occurs depends on how quickly the effusion grows and may be anywhere between a few hundred millilitres to several litres. The therapeutic consequence of the different haemodynamics of acute versus chronic tamponade is that, in the former, removal of $100 \mathrm{ml}$ of fluid can drop pericardial pressure dramatically and be life saving, whereas in the latter, considerably more fluid must be removed to attain comparable relief of tamponade. Between these extremes is a broad spectrum of pericardial volume and pressure.

\section{CLASSIC TAMPONADE}

Pericardial pressure, by definition, is elevated. The circulation adapts by increasing central systemic and pulmonary venous pressures to equal the pericardial pressure, thereby preventing total collapse of the cardiac chambers. Left and right ventricular diastolic, right atrial, and pulmonary wedge pressures all rise to equal the pressure in the pericardium, making atrial and ventricular diastolic transmural pressures essentially zero. This equalisation of pressures is the hallmark of tamponade. ${ }^{4}$ Stroke volume is reduced, but cardiac output is partially preserved by tachycardia, driven by increased adrenergic tone. The increased ventricular diastolic pressure mandates commensurate pulmonary hypertension to maintain pulmonary circulation. Pulmonary arterial systolic pressure seldom significantly exceeds $40 \mathrm{~mm} \mathrm{Hg}$.

When the pericardium is tightly stretched, it becomes virtually inextensible. As a result, total pericardial volume is constant throughout the respiratory cycle. Any change in the volume of one side of the heart then causes the opposite change in volume of the other side, a phenomenon referred to as ventricular interaction or interdependence. The manifestations of this greatly enhanced ventricular interaction are pulsus paradoxus ${ }^{45}$ and increased respiratory variation in the velocity of atrioventricular filling. ${ }^{6}$

Inspiration increases systemic venous return by the same mechanism as in normal physiology. The resulting increase in right heart volume can only occur by bulging the atrial and ventricular septa into the left atrium and ventricle. This redistribution of blood volume within the heart accompanying each respiratory cycle is the cause of decreased left ventricular stroke input and output and systemic arterial systolic pressure (pulsus paradoxus). Pulsus paradoxus can be palpated in the peripheral pulses, or measured by sphygmomanometry, or by cannulating a systemic artery. Respiratory changes in the pulmonary circulation are nearly $180^{\circ}$ out of phase with those in the systemic circulation as a consequence of ventricular interaction. ${ }^{5}$

\section{Severity spectrum}

Incipient or preclinical tamponade exists when pericardial pressure equals the normal right, but remains lower than left atrial pressure. When pericardial pressure rises to the level of normal right atrial pressure, cardiac tamponade is trivial; when it equals normal left atrial pressure, tamponade is mild. When pericardial pressure increases further, it reaches left atrial pressure. When pericardial pressure exceeds 10$12 \mathrm{~mm} \mathrm{Hg}$, moderate tamponade is present ${ }^{7}$ and detectable at the bedside by the abnormal jugular pulse and echocardiography that shows compression of the right heart chambers. ${ }^{8}$ The outer wall of the right atrium becomes concave (right atrial compression) ${ }^{9}$ and the right ventricular free wall indents toward the septum (right ventricular diastolic collapse) due to transient reversal of the transmural ventricular diastolic pressure. ${ }^{10}$ At this stage, pulsus paradoxus is often absent and the blood pressure and cardiac output are only slightly lowered. Increased respiratory 
variation of ventricular filling velocities is more pronounced. This phenomenon is detected by Doppler interrogation of ventricular inflow. For ease of measurement, the change in peak velocity, not velocity-time index or stroke volume, is traditionally used. When tamponade is severe (pericardial pressure more than $25 \mathrm{~mm} \mathrm{Hg}$ ), pulsus paradoxus is prominent, stroke volume and blood pressure are significantly reduced, and sinus tachycardia is usual. With increasing severity of tamponade, haemodynamics decline to the point of shock and, if not promptly treated, death.

\section{ATYPICAL TAMPONADE Cardiac co-morbidity}

It is important to bear in mind that pre-existing heart disease modifies the classic haemodynamics of tamponade. Conditions that greatly increase left ventricular diastolic pressure, atrial septal defect, and aortic regurgitation prevent pulsus paradoxus, even in severe tamponade. ${ }^{4}$ Patients with chronic renal disease and pericardial effusion therefore often fail to show pulsus paradoxus. ${ }^{7}$ Right heart failure or right ventricular hypertrophy prevents compression of right heart chambers.

Loculated effusion may cause regional tamponade. ${ }^{11}$ When the atria and great veins are spared, the features of tamponade are less for a given elevation of pericardial pressure. ${ }^{12}$ Regional tamponade found after cardiac surgery may compress the left ventricle instead of the right. ${ }^{13}$

\section{Low pressure tamponade}

While central venous pressure is typically elevated in proportion to tamponade, hypovolaemia may keep the central venous pressure low in spite of significant tamponade. ${ }^{14}$ In tamponade secondary to trauma, the explanation is exsanguination. In other cases the cause may be excessive diuretic treatment, often given in the belief that heart failure was the cause of raised central venous pressure. The most troublesome example is haemodialysis. If these patients are undertreated, the effusion may disappear after increased frequency of dialysis. On the other hand dialysis may have induced hypovolaemia, in which case the patient requires volume infusion.

\section{Chronic effusive pericarditis}

These massive effusions may persist for years. Less commonly they are recurrent. ${ }^{15}$ They accumulate slowly, accounting for their huge volume. The pericardium has stretched gradually, therefore pericardial pressure is only mildly elevated. The cases meet the criteria for preclinical or mild tamponade, which of itself does not require treatment. A significant proportion of the cases unpredictably develop classic tamponade, which is the reason for pericardiocentesis, and when that is followed by recurrences, pericardiectomy is recommended. ${ }^{15}$

\section{Effusive constrictive pericarditis}

Here, cardiac tamponade develops in the setting of a scarred pericardium causing constriction. Common causes are prior radiation and neoplasm. ${ }^{2}$

Less commonly, this syndrome follows acute pericarditis, in which the constriction may be transient. ${ }^{16}$ Pulsus paradoxus is less common than in classic tamponade and the central venous pressure often shows a prominent $y$ descent, whereas this descent is absent in classic tamponade. When haemodynamics are measured during pericardiocentesis, elevation of the central and pulmonary venous pressures persists after pericardial pressure has been reduced to normal.

Correspondence to: Ralph Shabetai, MD, VA Health Care System, Cardiology 111 A, La Jolla, CA 92161, USA; rshabetai@ucsd.edu

\section{REFERENCES}

1 Holt JP, Rhode EA, Kines H. Pericardial and ventricular pressure. Circ Res 1960:8:1171-81.

2 Sagrista-Sauleda J, Angel J, Permanyer-Miralda G, et al. Long-term follow-up of idiopathic chronic pericardial effusion. N Engl J Med 1999:341:2054-9.

3 LeWinter MM, Pavelec R. Influence of the pericardium on left ventricular end-diastolic pressure-segment relations during early and late stages of experimental chronic volume overload in dogs. Circ Res 1982;50:501-9.

4 Shabetai R, Fowler NO, Guntheroth WG. The hemodynamics of cardiac tamponade and constrictive pericarditis. Am J Cardiol 1970;26:480-9.

5 Shabetai R, Fowler NO, Fenton JC, et al. J Clin Invest 1965;44:1882-98.

6 Appleton CP, Hatle LK, Popp RL. Cardiac tamponade and pericardial effusion: respiratory variation in transvalvular flow velocities studied by Doppler echocardiography. J Am Coll Cardiol 1988;11:1020-30.

7 Reddy PS, Curtiss El, O'Toole JD, et al. Cardiac tamponade: hemodynamic observations in man. Circulation 1978;58:265-72.

8 Klopfenstein HS, Cogswell TL, Bernath GA, et al. Alterations in intravascular volume affect the relation between right ventricular diastolic collapse and the hemodynamic severity of cardiac tamponade. J Am Coll Cardiol 1985:6:1057-63.

9 Gillam LD, Guyer DE, Gibson TC, et al. Hydrodynamic compression of the right atrium: a new echocardiographic sign of cardiac tamponade. Circulation 1983;68:294-301.

10 Leimgruber PP, Klopfenstein HS, Wann LS, et al. The hemodynamic derangement associated with right ventricular diastolic collapse in cardiac tamponade: an experimental echocardiographic study. Circulation 1983;68:612-20.

11 Fowler NO, Gabel M. Regional cardiac tamponade: a hemodynamic study. $J$ Am Coll Cardiol 1987; 10:164-9.

12 Fowler NO, Gabel M. The haemodynamic effects of cardiac tamponade: mainly the result of atrial, not ventricular, compression. Circulation 1985;71:154-7.

13 Chuttani K, Pandian NG, Mohanty PK, et al. Left ventricular diastolic collapse. An echocardiographic sign of regional cardiac tamponade. Circulation 1991;83:1999-2006

14 Hayes SN, Freeman WK, Gersh BJ. Low pressure cardiac tamponade: diagnosis facilitated by Doppler echocardiography. Br Heart $J$ 1990;63:136-40.

15 Hancock EW. Subacute effusive-constrictive pericarditis. Circulation 1971;43:183-92.

16 Sagrista-Sauleda J, Permanyer-Miralda G, Candell-Riera J, et al. Transient cardiac constriction: an unrecognized pattern of evolution in effusive acute idiopathic pericarditis. Am J Cardiol 1987;59:961-6. 